\title{
A Test Point Selection Method Based on Circuit Topology Graph
}

\author{
DUAN Xiusheng \\ Department of Electronic and Optical Engineering \\ Ordnance Engineering College \\ Shijiazhuang, China \\ sjzdxsh@163.com
}

\author{
HUANG Yi \\ Department of Electronic and Optical Engineering \\ Ordnance Engineering College \\ Shijiazhuang, China \\ dd_huangyi@163.com
}

\author{
ZHOU Yunfeng \\ General Armaments Department \\ Chongqing Military Representative Bureau \\ Chongqing, China \\ zyf7962@163.com
}

\begin{abstract}
To the question of test point selection in test or diagnosis for complex electronic equipments and large scale circuit, a test point selection method based on circuit topology and correlation modeling is proposed. This method, firstly, established the topology model based on circuit function structure, then the adjacency matrix of module connection relationship in reaction model and get the initial point set; secondly established correlation modeling using adjacency matrix; then, traversal correlation matrix and delete redundant test point based on reverse deletion strategy, obtain optimal test set; at last, verify this method with a example.
\end{abstract}

Keywords-circuit; topology structure; test point; correlation matrix; optimal test set.

\section{INTRODUCTION}

The optimal test point selection plays an important role in electronic equipment test and fault diagnosis, the purpose of which is to select the test point subset covers all faults and costs (time, fee etc.) little, namely optimal test set. With the scale and complexity of electronic equipment growing, the test point selection becomes more and more important in reducing cost of equipment test and diagnosis and improving testability design, and has already become a hotspot and difficulty of current research.

\section{THE ESTABLISHMENT OF CORRELATION MODEL BASED ON CIRCUIT TOPOLOGY STRUCTURE}

\section{A. The establishment of circuit topology structure}

For test and diagnosis in large scale and complex electronic equipment, generally, firstly classify the equipments into four levels according to function and structure: system level, subsystem level, unit level and circuit board level, then do test and diagnosis level by level; for complex circuit, usually divide the circuit into several modules first, then do test and diagnosis. According to the function module and physical structure, divide the test object into several independent unit or module (hereinafter generally referred to as module). Based on this, utilize these modules as test nodes and establish the directed topology graph of test object according to their connection relationship. When a module has more than one mutually independent outputs, decompose it into several single output module to ensure that every module has only one output in the topology graph and normal and abnormal state are indicated by output[1]. Fig. 1 is a circuit topology contains 4 single output modules, in which $M_{i}$ represents module $i$. The initial default test point is the output set.

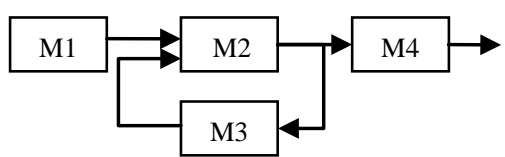

Fig. 1. A circuit topology composed of 4 single output modules

\section{B. The establishment of correlation module}

Based on the circuit topology graph in Fig. 1, establish the adjacency matrix of these modules. The procedure is: if directed line segment comes from $M_{i}$ and go to $M_{j}$, the element $a_{i j}$ in adjacency matrix $A$ is 1 . Get the results as below:

$$
A=\left[\begin{array}{llll}
1 & 1 & 1 & 1 \\
0 & 1 & 1 & 1 \\
0 & 1 & 1 & 1 \\
0 & 0 & 0 & 1
\end{array}\right]
$$


$\square$ The correlation module is represented with correlation matrix. Correlation matrix also use 1,0 as elements, which represents two nodes are correlated or uncorrelated. The hypothesis of correlation modeling method could be described as below [2]:

1) The object under test only has two states, normal or fault; when there is no fault, the object works normal.

2) When the measured object is in a state of failure, assuming that only one module fault happens, that is the single-fault assumption. In fact, though the measured object has 2 different faults at the same time (probability is very low), they can be isolated individually in real diagnosis.

3) The state of measured object completely depends on the states of every module. If anyone module is fault, it will affect the other module's test points that the signal can reach, so the effectiveness of all these test points is the same.

According to Warshall algorithm, we can get correlation matrix $P=\left(p_{i j}\right)$ directly by adjacency matrix. The procedure of the algorithm is as below [3]:

a) set new matrix $P=A$;

b) set $k=1$;

c) for all of $i$, if $P(i, k)=1$, act $P(i, j)=P(i, j) \vee P(k, j)$ for $j=1 \cdots n$, where “ $\vee$ ” is Boolean sum, namely “or” operation;

d) $k+1$;

$e)$ if $k \leq n$, turn to c), or stop;

$f)$ change all elements value at diagonal to 1 .

For the circuit in Fig. 1, under single fault assumption, there can be only 4 fault states. Suppose that $F_{i}$ is fault state corresponding to $B_{i}$, and $T_{i}$ is test point corresponding to $B_{i}$, where $i=1 \sim 4$. By calculating, the correlation matrix $P$ corresponding to Fig. 1 could be obtained:

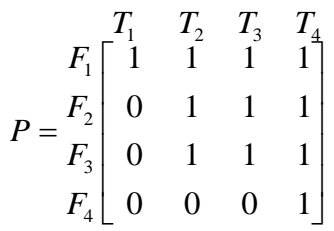

Where column sign of matrix represents test points and row sign of matrix represents faults. So the relationships between the circuit's test points and faults can be represented by the correlation matrix $P[4]$.

\section{TEST POINT SELECTION BASED ON REVERSE DELETION STRATEGY}

After getting the correlation between test point and fault, it can be applied to test point selection. To reduce subsequent workload, it needs to be simplified first. The detail procedure is as below [5]:

a) Comparing columns of matrix, if column vector $T_{x}=T_{y}(x \neq y)$, the test point is redundant to each other and choosing one of them is ok, namely delete the corresponding column to the test point in the matrix;

b) Comparing rows of the matrix, if row vector $F_{x}=F_{y}(x \neq y)$, the two corresponding faults can not be classified and should be addressed as one fault, namely one row of them is enough[6,7].

After simplification, part of the redundant test point has been deleted. Suppose that all test points cost the same, so we can delete other redundant test points using correlation matrix segmentation. In this method, the number of segmentation matrixes show exponential growth with the number of test point, so under the condition of complex test object and a lot of test points, the procedure to select optimal test point will be complex and time-long.

On selecting test point, positive selection strategy can be utilized, namely select test point need to be saved one by one and obtain the optimal test point set finally; as well as reverse deletion strategy, select test point can be deleted one by one and remove these points in initial test point set, namely obtain the optimal test point set.

When the initial test point set is some large, generally, using positive selection strategy is complex, so reverse deletion strategy is usually utilized. Firstly, select possible redundant test point from initial test point set, which is relatively small, and then selectively delete these test points one by one. According to the feature of redundant test point, the test point can be deleted meets the following properties:

a) After the column vector corresponding to test point can be deleted being deleting from the correlation matrix, the rest matrix has no row vector with all zero.

b) After the column vector corresponding to test point can be deleted being deleting from the correlation matrix, there is no same row in the rest matrix [8].

In this method, firstly, search and get the initial test point set through one time traversal; then traverse $T_{d}$ with any start test point $i$ in $T_{4} \beta \underline{=} \underset{\chi}{ }$. in the same time; finally, find the set $T_{d \max }$ that contains the most test point from all of $T_{d i}$ and delete $T_{d \max }$ from the initial test point set, namely get the optimal test point set.

\section{EXPERIMENTAL VERIFICATION}

Choose a fire control system as study object, which is composed of 7 units. These units are the power supply system, operating rod, track electronic box, fire control computers, stable tracking computers, gun control and servo system, etc. The 7 units can also be divided into 16 modules. And then we can establish its ciruit topology graph, which is according to the 7 units or 16 modules' function, as shown in Fig. 2. 


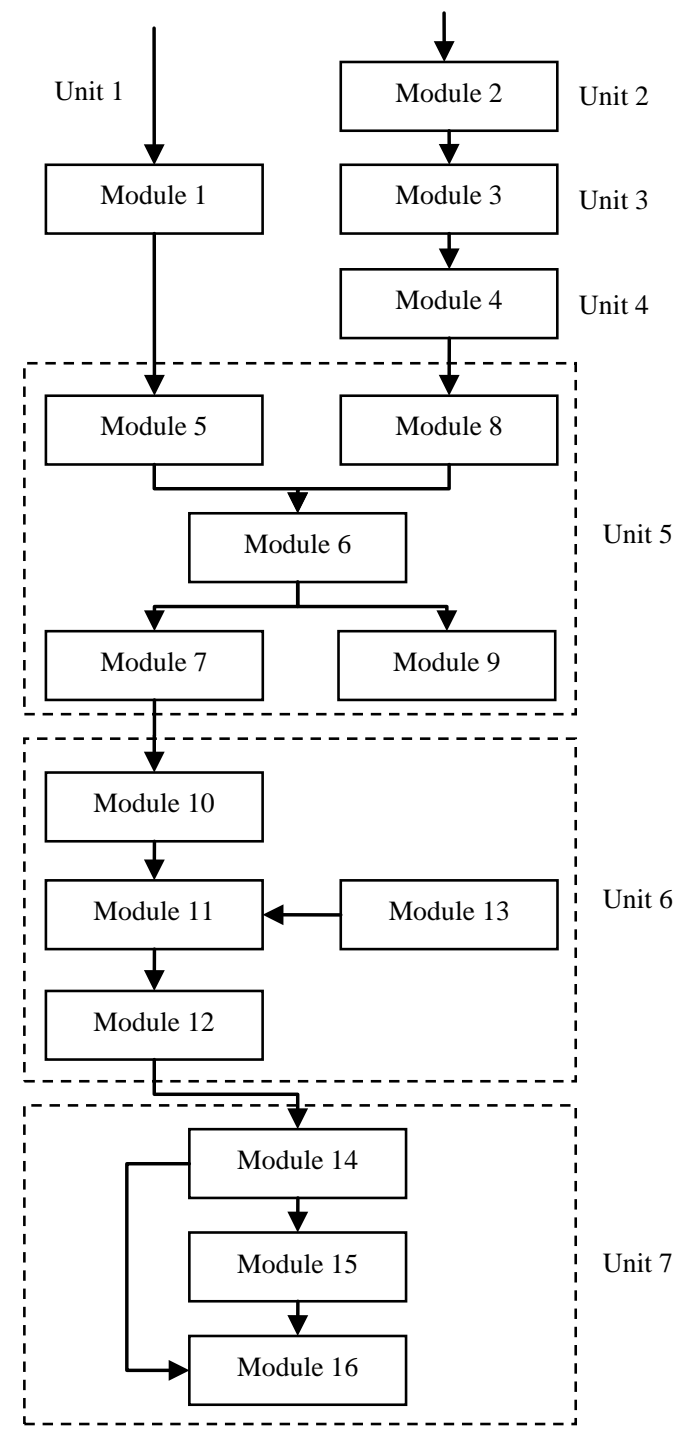

Fig. 2. The circuit topology graph of a fire control system

According to the topology structure of system and connection relationship between modules, get adjacency matrix $A[9,10]$ :
According to Warshall algorithm, calculate and get the correlation matrix $P$ of system[11]:

$$
P=\left[\begin{array}{l}
1,0,0,0,1,1,1,0,1,1,1,1,0,1,1,1 \\
0,1,1,1,0,1,1,1,1,1,1,1,0,1,1,1 \\
0,0,1,1,0,1,1,1,1,1,1,1,0,1,1,1 \\
0,0,0,1,0,1,1,1,1,1,1,1,0,1,1,1 \\
0,0,0,0,1,1,1,0,1,1,1,1,0,1,1,1 \\
0,0,0,0,0,1,1,0,1,1,1,1,0,1,1 \\
0,0,0,0,0,0,1,0,0,1,1,1,0,1,1,1 \\
0,0,0,0,0,1,1,1,1,1,1,1,0,1,1,1 \\
0,0,0,0,0,0,0,0,1,1,1,1,0,1,1,1 \\
0,0,0,0,0,0,0,0,0,1,1,1,0,1,1,1 \\
0,0,0,0,0,0,0,0,0,0,1,1,0,1,1,1 \\
0,0,0,0,0,0,0,0,0,0,0,1,0,1,1,1 \\
0,0,0,0,0,0,0,0,0,0,1,1,1,1,1,1 \\
0,0,0,0,0,0,0,0,0,0,0,0,0,1,1,1 \\
0,0,0,0,0,0,0,0,0,0,0,0,0,0,1,1 \\
0,0,0,0,0,0,0,0,0,0,0,0,0,0,0,1
\end{array}\right]
$$

According to the reverse delete strategy, on the basis of the principles putforward above, firstly, select the initial set of test points can be deleted $T_{d}=\{7,9,15\}$; then traverse $T_{d}$ with each test point in $T_{d}$ as starting point, find all sets of test points can be deleted and get the max one $T_{d \max }=\{7,15\}$; finally, the initial test points set subtracts set $T_{d \max }$ and get the optimal test points set $\{1,2,3,4,5,6,8,9,10,11,12,13,14,16\}$.

\section{CONCLUSION}

Aiming at test and diagnosis of complex electronic equipment and large scale circuit, we analyzed the test point selection algorithm, put forward a test point selection method based on circuit topology structure and correlation model and simplified the algorithm using reverse deletion strategy. This method has reference value in increasing test efficiency and reducing test complexity in equipment technique support.

\section{REFERENCES}

[1] MAO Donghui, ZHOU Yuewen, and CHEN Ziliang et. al., "The Research on Optimizing Test Point for Air-to-air Missile System,” J. Journal of Projectiles, Rockets, Missiles and Guidance, vol.1 pp.71-74, 2011. (in Chinese)

[2] Li Guangshen, Chen Yongwei, and Xie Yongcheng, "Design for Testnode and Diagnosis Strategy of the Armored Vehicle's Electric System," J. Ship Electronic Engineering. vol.5 pp. 142-145, 2011. (in Chinese)

[3] DING Hao, "Modeling, Analysis and Software Design for System Testability Based on Muti-signal Flow Graph,” D. Harbin Institute of Technology, 2014. (in Chinese)

[4] ZHANG Shigang, "Research on the Optimization and Generation Techniques for Diagnostic Strategy Based on Multi-signal Model,” D. National Univefsigy of Defense T\&qhyology, 2py0. (in Chinese)

[5] MAO Donghui, ZHOU Yuewen, and CHEN Ziliang et. al., "The Research on Optimizing Test Point for Air-to-air Missile System,” J. Journal of Projectiles, Rockets, Missiles and Guidance, vol.1 pp.71-74, 2011. (in Chinese)

[6] Zhang Zhi, Wang Weiping, and Li Yu, "Optimal Diagnosis Strategy of Built-in Test Based on Information Entropy,” J. Computer Measurement \& Control. vol. 7 pp. 1744-1746, 2012

[7] Yang Zhiyong, Xu Hualong, and Xu Aiqiang, "Design of Diagnosis Strategy Based on Multisignal Model,” J. Computer Measurement \& Control. vol. 12 pp. 1616-1619, 2006. 
[8] XU Jun, CHANG Tian-qing, and GUO You-song et. al., “Optimal Design of Test Point Based on the Maximum Failure Feature Information Entropy,” J. Journal of Armored Force Engineering Institute. vol. 3 pp. 41-44, 2009.

[9] ZHOU Yan, ZHOU Bu-xiang, and XING Yi, “Graphical power network topology analysis based on adjacency matrix,” J. Power System Protection and Control. vol. 17 pp. 49-52, 2009.
[10] Wei Jicheng, "Determination of Network Topology by Matrix Partial Multiplication,” D. Dalian Maritime University,2012.

[11] WANG Ziling, XU Aiqiang, and NIU Shuangcheng, "A New Method for Building Dependency Matrix of Complex System,” J. Journal of Test and Measurement Technology. vol. 2 pp. 182-188, 2010. 